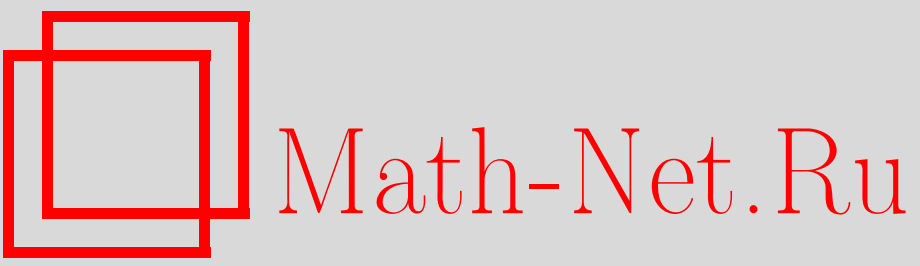

С. Н. Асхабов, Нелинейные интегральные уравнения с ядрами типа потенциала в непериодическом случае, Итоги науки и техн. Сер. Соврем. мат. и ее прил. Темат. обз., 2019, том 170, 3-14

DOI: https://doi.org/10.36535/0233-6723-2019-170-3-14

Использование Общероссийского математического портала Math-Net.Ru подразумевает, что вы прочитали и согласны с пользовательским соглашением

http://www.mathnet.ru/rus/agreement

Параметры загрузки:

IP: 18.208 .226 .222

26 апреля 2023 г., $12: 48: 14$ 


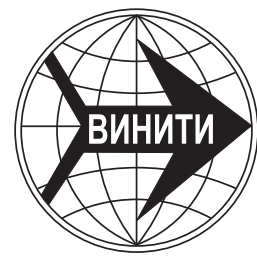

ИТОГИ НАУКИ И ТЕХНИКИ.

Современная математика и ее приложения.

Тематические обзоры.

Том 170 (2019). С. 3-14

DOI: $10.36535 / 0233-6723-2019-170-3-14$

УДК 517.968.4

\title{
НЕЛИНЕЙНЫЕ ИНТЕГРАЛЬНЫЕ УРАВНЕНИЯ С ЯДРАМИ ТИПА ПОТЕНЦИАЛА В НЕПЕРИОДИЧЕСКОМ СЛУЧАЕ
}

\author{
(c) 2019 г. $\quad$ C. H. АСХАБОВ
}

\begin{abstract}
АннотАция. Найдены условия при которых обобщенный оператор типа потенциала действует непрерывно из пространства Лебега с весом общего вида в сопряженное с ним пространство и обладает свойством положительности. Используя эти условия, методом монотонных (по БраудеруМинти) операторов доказаны глобальные теоремы о существовании и единственности решения для различных классов нелинейных интегральных уравнений типа свертки в вещественных весовых пространствах Лебега. Получены оценки норм решений, из которых следует, что соответствующие однородные уравнения имеют лишь тривиальное решение.
\end{abstract}

Ключевые слова: положительный оператор, обобщенный оператор типа потенциала, монотонный оператор, нелинейное интегральное уравнение.

\section{NONLINEAR INTEGRAL EQUATIONS WITH POTENTIAL-TYPE KERNELS IN THE NONPERIODIC CASE}

\author{
(c) 2019 S. N. ASKHABOV
}

\begin{abstract}
We find conditions under which a generalized potential-type operator acts continuously from a Lebesgue space with a general weight to its dual space and possesses the positivity property. Using these conditions, the global existence and uniqueness theorems for various classes of nonlinear integral equations of convolution type in real weighted Lebesgue spaces are proved by the method of monotonic (in the Browder-Minty sense) operators. Also we obtain estimates of the norms of solutions which imply that the corresponding homogeneous equations have only a trivial solution.
\end{abstract}

Keywords and phrases: positive operator, generalized potential-type operator, monotonic operator, nonlinear integral equation.

AMS Subject Classification: 45G10, 47J05

1. Введение. В настоящее время теория линейных уравнений типа свертки достаточно хорошо разработана (см., например, [8]). В монографии [1] изучены различные классы нелинейных интегральных уравнений типа свертки как на конечном (в периодическом случае), так и на бесконечном промежутке интегрирования. Существенную роль при этом играет свойство положительности (по Бохнеру) интегрального оператора типа свертки, которое обеспечивается условием неотрицательности дискретного (в случае отрезка $[-\pi, \pi]$ ) или интегрального (в случае оси $\mathbb{R}$ ) косинус-преобразования Фурье его ядра. В случае отрезка $[-\pi, \pi]$ примерами таких ядер могут служить некоторые выпуклые вниз функции. В рассматриваемом здесь случае отрезка $[0,1]$ при исследовании нелинейных интегральных уравнений с ядрами типа потенциала возникают дополнительные трудности, связанные по сути дела с тем, что для положительности оператора типа

Работа выполнена при поддержке Российского фонда фундаментальных исследований (проект № 18-41-200001). 
свертки выпуклости вниз его ядра уже не достаточно. При дополнительных ограничениях на ядра рассматриваемых уравнений, в данной работе методом монотонных (по Браудеру-Минти) операторов доказаны глобальные теоремы о существовании, единственности и оценках решений для различных классов нелинейных интегральных уравнений с ядрами типа потенциала в вещественных пространствах Лебега $L_{01}^{p}(\rho)$ с произвольным (не обязательно степенным) весом $\rho(x)$ при любых $p \in(1, \infty)$. Ранее подобные теоремы были доказаны, в зависимости от рассматриваемого класса нелинейных уравнений, либо только при $p \in(1,2]$, либо только при $p \in[2, \infty)$ (см. [1, гл. III]). В отличие от $[6,7,12,13]$ и других работ, наше исследование нелинейных уравнений проводится без предположения о том, что линейный интегральный оператор (в данном случае обобщенный оператор типа потенциала) заведомо является положительным, а также либо не использует, как в [12,13], условие коэрцитивности оператора Немыцкого $F$ (теорема 3.3), либо основано на обращении этого оператора и установлении коэрцитивности обратного к нему оператора $F^{-1}$ (теорема 3.4).

Метод монотонных (по Браудеру-Минти) операторов (см., например, монографии [6,7] и приведенную в них библиографию) хорошо известен применительно к нелинейным интегральным уравнениям Гаммерштейна (именно к ним он впервые был применен). Поскольку интегральные уравнения Гаммерштейна имеют ядро общего вида (функция двух переменных), то они изучались в предположении, что линейный интегральный оператор с этим ядром априори является положительным. В случае нелинейных интегральных уравнений типа свертки естественно возникает задача нахождения условий на ядро (функция одной переменной), при которых линейные интегральные операторы свертки являются положительными. Решение этой задачи представляет интерес и для современного гармонического анализа, в котором основополагающую роль играет понятие положительно определенной (по Бохнеру) функции (см. [11]), тесно связанное с понятием положительного оператора. В этой связи в данной работе доказаны теоремы о положительности обобщенного оператора типа потенциала, охватывающие, в частности, потенциал Рисса и логарифмический потенциал. Эти теоремы дополняют результаты, полученные в [4,9]. Следует отметить, что теория нелинейных интегральных уравнений с разностными ядрами достаточно хорошо разработана в вольтерровском случае (см. $[14,15])$. Что касается рассматриваемых нами уравнений, то они сравнительно мало изучены и их исследование имеет не только теоретическое, но и прикладное значение в связи с приложениями в теории следящих систем и электрических сетей, в эпидемической и генетической моделях и др. (см., например, монографию [1] и приведенную в ней библиографию).

Всюду в работе будем придерживаться принятых в монографии [7] обозначений и определений, касающихся теории монотонных операторов. Пусть $X$-вещественное рефлексивное банахово пространство, $X^{*}$ - сопряженное с ним пространство и оператор $A$ действует из $X$ в $X^{*}$. Обозначим через $\langle y, x\rangle$ значение линейного непрерывного функционала $y \in X^{*}$ на элементе $x \in X$. В частности, если $X$ - это гильбертово пространство $H$, то $\langle y, x\rangle$ совпадает со скалярным произведением $(y, x)$, где $x, y \in H$. Оператор $A$ называется монотонным, если для любых $u, v \in X$ выполняется неравенство $\langle A u-A v, u-v\rangle \geqslant 0$. Если $A$-линейный оператор, то определение монотонного оператора совпадает с определением положительного оператора.

Как обычно, через $\mathbb{R}$ и $\mathbb{N}$ обозначаются, соответственно, множества всех действительных и натуральных чисел, а через $p^{\prime}=p /(p-1)$ - сопряженное с $p$ число $\left(1 / p+1 / p^{\prime}=1\right)$.

2. Положительность обобщенного оператора типа потенциала. Пусть $\rho(x)-$ неотрицательная почти всюду конечная и почти всюду отличная от нуля измеримая по Лебегу на отрезке $[0,1]$ функция. Обозначим через $L_{01}^{p}(\rho), 1 \leqslant p<\infty$, множество всех измеримых по Лебегу на отрезке $[0,1]$ функций $u(x)$ с конечной нормой

$$
\|u\|_{p, 1}=\left(\int_{0}^{1} \rho(x) \cdot|u(x)|^{p} d x\right)^{1 / p} .
$$

Известно (см., например, [1]), что $L_{01}^{p}(\rho)$ при $1<p<\infty$ является рефлексивным банаховым пространством, а сопряженным с ним является пространство $L_{01}^{p^{\prime}}\left(\rho^{1-p^{\prime}}\right)$, где $p^{\prime}=p /(p-1)$. Пространство $L_{01}^{p}(\rho)$ называют весовым пространством Лебега с весом $\rho(x)$. Если $\rho(x)=1$, 
то будем писать $L_{01}^{p}$ и $\|\cdot\|_{p}$, соответственно. Норму в пространстве $L_{01}^{p^{\prime}}\left(\rho^{1-p^{\prime}}\right)$ будем обозначать через $\|\cdot\|_{p^{\prime}, 1-p^{\prime}}$.

Следующие три теоремы будут играть важную роль при исследовании далее трех различных классов нелинейных интегральных уравнений с ядрами типа потенциала.

Теорема 2.1. Пусть $1<p<\infty$ и вес $\rho(x)$ удовлетворяет следующим условиям:

$$
\begin{aligned}
& \left\|\rho^{-1}\right\|_{\infty}^{1 / p}=\left[\operatorname{esssup}_{0 \leqslant x \leqslant 1} \rho^{1-p^{\prime}}(x)\right]^{1 / p^{\prime}}<\infty, \quad \text { если } 1<p \leqslant 2, \\
& \left\|\rho^{-1}\right\|_{2 /(p-2)}^{1 / p}=\left(\int_{0}^{1}[\rho(x)]^{2 /(2-p)} d x\right)^{(p-2) /(2 p)}<\infty, \quad \text { если } 2<p<\infty .
\end{aligned}
$$

Если $\varphi(x) \in L_{01}^{p^{\prime} / 2}$ при $1<p \leqslant 2$ или $\varphi(x) \in L_{01}^{1}$ при $2<p<\infty$, то обобщенный оператор типа потенциала

$$
\left(P_{01}^{\varphi} u\right)(x)=\int_{0}^{1} \varphi(|x-t|) \cdot u(t) d t
$$

действует непрерывно из $L_{01}^{p}(\rho)$ в $L_{01}^{p^{\prime}}\left(\rho^{1-p^{\prime}}\right)$, причем для любого $u(x) \in L_{01}^{p}(\rho)$ выполняется неравенство

$$
\left\|P_{01}^{\varphi} u\right\|_{p^{\prime}, 1-p^{\prime}} \leqslant C \cdot\|u\|_{p, 1}
$$

¿əe

$$
C= \begin{cases}2^{2 / p^{\prime}}\left\|\rho^{-1}\right\|_{\infty}^{2 / p}\|\varphi\|_{p^{\prime} / 2}, & \text { если } 1<p \leqslant 2 \\ 2\left\|\rho^{-1}\right\|_{2 /(p-2)}^{2 / p}\|\varphi\|_{1}, & \text { если } 2<p<\infty\end{cases}
$$

Доказательство. Рассмотрим отдельно два случая.

1. Пусть $1<p \leqslant 2$. Тогда

$$
A=\underset{0 \leqslant t \leqslant 1}{\operatorname{ess} \sup _{0}}\left(\int_{0}^{1} \rho^{1-p^{\prime}}(x) \cdot|\varphi(|x-t|)|^{p^{\prime} / 2} d x\right)^{1 / p^{\prime}}<\infty
$$

В самом деле, так как для любого $t \in[0,1]$ имеем

$$
\begin{aligned}
\int_{0}^{1}|\varphi(|x-t|)|^{p^{\prime} / 2} d x=\int_{0}^{t}|\varphi(t-x)|^{p^{\prime} / 2} d x+\int_{t}^{1}|\varphi(x-t)|^{p^{\prime} / 2} d x= \\
\quad=\int_{0}^{t}|\varphi(s)|^{p^{\prime} / 2} d s+\int_{0}^{1-t}|\varphi(s)|^{p^{\prime} / 2} d s \leqslant 2 \int_{0}^{1}|\varphi(s)|^{p^{\prime} / 2} d s=2\|\varphi\|_{p^{\prime} / 2}^{p^{\prime} / 2}
\end{aligned}
$$

то, в силу условия (2.1),

$$
A \leqslant 2^{1 / p^{\prime}}\left\|\rho^{-1}\right\|_{\infty}^{1 / p}\|\varphi\|_{p^{\prime} / 2}^{1 / 2}
$$


Докажем оценку (2.3). Применяя неравенство Гельдера и учитывая, что $-p^{\prime} / p=1-p^{\prime}$, имеем

$$
\begin{aligned}
\left|\left(P_{01}^{\varphi} u\right)(x)\right| \leqslant & \int_{0}^{1} \rho^{-1 / p}(t)|\varphi(|x-t|)|^{1 / 2} \rho^{1 / p}(t)|\varphi(|x-t|)|^{1 / 2}|u(t)| d t \leqslant \\
\leqslant & \left(\int_{0}^{1} \rho^{-p^{\prime} / p}(t)|\varphi(|x-t|)|^{p^{\prime} / 2} d t\right)^{1 / p^{\prime}}\left(\int_{0}^{1} \rho(t)|\varphi(|x-t|)|^{p / 2}|u(t)|^{p} d t\right)^{1 / p} \leqslant \\
& \leqslant A \cdot\left(\int_{0}^{1} \rho(t)|\varphi(|x-t|)|^{p / 2}|u(t)|^{p} d t\right)^{1 / p} .
\end{aligned}
$$

Следовательно,

$$
\begin{aligned}
& \left\|P_{01}^{\varphi} u\right\|_{p^{\prime}, 1-p^{\prime}}=\left(\int_{0}^{1} \rho^{1-p^{\prime}}(x)\left|\left(P_{01}^{\varphi} u\right)(x)\right|^{p^{\prime}} d x\right)^{1 / p^{\prime}} \leqslant \\
& \leqslant A\left(\int_{0}^{1} \rho^{1-p^{\prime}}(x)\left(\int_{0}^{1} \rho(t)|\varphi(|x-t|)|^{p / 2}|u(t)|^{p} d t\right)^{p^{\prime} / p} d x\right)^{1 / p^{\prime}}= \\
& =A\left[\left(\int_{0}^{1}\left(\int_{0}^{1} \rho^{\left(1-p^{\prime}\right) p / p^{\prime}}(x) \rho(t)|\varphi(|x-t|)|^{p / 2}|u(t)|^{p} d t\right)^{p^{\prime} / p} d x\right)^{p / p^{\prime}}\right]^{1 / p} \leqslant 1 \\
& \leqslant A\left[\int_{0}^{1}\left(\int_{0}^{1}\left(\rho^{-1}(x) \rho(t)|\varphi(|x-t|)|^{p / 2}|u(t)|^{p}\right)^{p^{\prime} / p} d x\right)^{p / p^{\prime}} d t\right]^{1 / p}= \\
& =A\left[\int_{0}^{1} \rho(t)|u(t)|^{p}\left(\int_{0}^{1} \rho^{1-p^{\prime}}(x)|\varphi(|x-t|)|^{p^{\prime} / 2} d x\right)^{p / p^{\prime}} d t\right]^{1 / p} \leqslant A^{2}\|u\|_{p, 1} .
\end{aligned}
$$

Поскольку, в силу (2.5),

$$
A^{2} \leqslant 2^{2 / p^{\prime}}\left\|\rho^{-1}\right\|_{\infty}^{2 / p}\|\varphi\|_{p^{\prime} / 2}
$$

то из предыдущего неравенства получаем доказываемую оценку (2.3).

2. Пусть, наконец, $2<p<\infty$. В этом случае, в силу условия $(2.2)$, имеют место непрерывные вложения

$$
L_{01}^{p}(\rho) \subset L_{01}^{2} \subset L_{01}^{p^{\prime}}\left(\rho^{1-p^{\prime}}\right),
$$

причем выполняются неравенства

$$
\begin{array}{ll}
\|u\|_{p^{\prime}, 1-p^{\prime}} \leqslant\left\|\rho^{-1}\right\|_{2 /(p-2)}^{1 / p}\|u\|_{2} & \text { для любого } u(x) \in L_{01}^{2}, \\
\|u\|_{2} \leqslant\left\|\rho^{-1}\right\|_{2 /(p-2)}^{1 / p}\|u\|_{p, 1} & \text { для любого } u(x) \in L_{01}^{p}(\rho) .
\end{array}
$$

Неравенство (2.7) доказывается применением неравенства Гельдера с показателями $2 /\left(2-p^{\prime}\right)$ и $2 / p^{\prime}$, а неравенство (2.8) - применением неравенства Гельдера с показателями $p /(p-2)$ и $p / 2$. Вложения (2.6) являются прямыми следствиями неравенств (2.7) и (2.8). Покажем предварительно,

\footnotetext{
${ }^{1}$ Здесь мы применили обобщенное неравенство Минковского с учетом того, что $p / p^{\prime} \geqslant 1$.
} 
что оператор $P_{01}^{\varphi}$ действует непрерывно из $L_{01}^{2}$ в $L_{01}^{2}$. Поскольку

$$
\begin{aligned}
\int_{0}^{1}|\varphi(|x-t|)| d t=\int_{0}^{x}|\varphi(x-t)| d t+\int_{x}^{1}|\varphi(t-x)| d t= & \\
& =\int_{0}^{x}|\varphi(s)| d s+\int_{0}^{1-x}|\varphi(s)| d s \leqslant 2\|\varphi\|_{1},
\end{aligned}
$$

то, применяя неравенство Коши-Буняковского, имеем

$$
\begin{aligned}
\left|\left(P_{01}^{\varphi} u\right)(x)\right| \leqslant \int_{0}^{1}|\varphi(|x-t|)|^{1 / 2}|\varphi(|x-t|)|^{1 / 2}|u(t)| d t \leqslant & \\
\leqslant\left(\int_{0}^{1}|\varphi(|x-t|)| d t\right)^{1 / 2} & \left(\left.\int_{0}^{1}|\varphi(|x-t|) \cdot| u(t)\right|^{2} \mid d t\right)^{1 / 2} \leqslant \\
& \leqslant \sqrt{2}\|\varphi\|_{1}^{1 / 2} \cdot\left(\left.\int_{0}^{1}|\varphi(|x-t|) \cdot| u(t)\right|^{2} \mid d t\right)^{1 / 2} .
\end{aligned}
$$

Используя сначала оценку (2.10), затем меняя порядок интегрирования (по теореме Фубини) и учитывая неравенство (2.9), получаем

$$
\begin{aligned}
\left\|P_{01}^{\varphi} u\right\|_{2} \leqslant \sqrt{2}\|\varphi\|_{1}^{1 / 2}\left[\int_{0}^{1}\left(\int_{0}^{1}|\varphi(|x-t|)| \cdot|u(t)|^{2} d t\right) d x\right]^{1 / 2}= \\
=\sqrt{2}\|\varphi\|_{1}^{1 / 2}\left[\int_{0}^{1}|u(t)|^{2}\left(\int_{0}^{1}|\varphi(|x-t|)| d x\right) d t\right]^{1 / 2} \leqslant 2\|\varphi\|_{1}\|u\|_{2},
\end{aligned}
$$

т.е. оператор $P_{01}^{\varphi}$ действует непрерывно из $L_{01}^{2}$ в $L_{01}^{2}$.

Докажем, наконец, что оператор $P_{01}^{\varphi}$ действует непрерывно из $L_{01}^{p}(\rho)$ в $L_{01}^{p^{\prime}}\left(\rho^{1-p^{\prime}}\right)$ и выполняется неравенство (2.3). Пусть $u(x) \in L_{01}^{p}(\rho)$ - произвольная функция. На основании первого вложения изЁ $(2.6)$ имеем, что $u(x) \in L_{01}^{2}$ и, значит, в силу $(2.11),\left(P_{01}^{\varphi} u\right)(x) \in L_{01}^{2}$. Но тогда, на основании второго вложения из $(2.6)\left(P_{01}^{\varphi} u\right)(x) \in L_{01}^{p^{\prime}}\left(\rho^{1-p^{\prime}}\right)$ и, в силу оценок $(2.7),(2.8)$ и $(2.11)$, справедливы неравенства

$$
\left\|P_{01}^{\varphi} u\right\|_{p^{\prime}, 1-p^{\prime}} \leqslant\left\|\rho^{-1}\right\|_{2 /(p-2)}^{1 / p}\left\|P_{01}^{\varphi} u\right\|_{2} \leqslant 2\|\varphi\|_{1}\left\|\rho^{-1}\right\|_{2 /(p-2)}^{1 / p}\|u\|_{2} \leqslant 2\left\|\rho^{-1}\right\|_{2 /(p-2)}^{2 / p}\|\varphi\|_{1}\|u\|_{p, 1} .
$$

Таким образом, неравенство (2.3) выполняется и в случае $2<p<\infty$.

Замечание 2.1. Так как $\left(1-p^{\prime}\right) / p^{\prime}=-1 / p$ при $p>1$ и $2 /(p-2)<1$ при $p>4$, то левые части равенств (2.1) и (2.2) следует понимать лишь как удобные обозначения правых частей этих равенств.

Далее нам понадобится следующая лемма, доказанная в [2] (ср. [10, с. 46]).

Лемма 2.1 (см. [2]). Если $f(x) \in C[0,1]$ - невозрастающая выпуклая вниз функиия, то для всех $n \in \mathbb{N}$ выполняется неравенство

$$
a_{n}=2 \int_{0}^{1} f(x) \cos (\pi n x) d x \geqslant 0,
$$

причем $a_{n}>0$, если $f(x)$ - строго выпуклая вниз убывающал функция. 
Следует отметить, что при более жестких ограничениях (неотрицательность и непрерывная дифференцируемость функции $f(x)$ ) лемма 2.1, в связи с приложениями в дробном (интегральном и дифференциальном) исчислении, была доказана другим путем в [9], где также доказано, что оператор $P_{01}^{\varphi}$ с ядром $\varphi(x)$, удовлетворяющим условию монотонности $\mathbf{M}$, т.е.

$$
\varphi(x) \in C^{1}(0,1], \quad \varphi(x) \geqslant 0, \quad \int_{0}^{1} \varphi(x) d x<\infty, \quad \varphi\left(x_{1}\right) \geqslant \varphi\left(x_{2}\right), \quad \varphi^{\prime}\left(x_{1}\right)<\varphi^{\prime}\left(x_{2}\right) \quad \forall x_{1}<x_{2},
$$

является строго положительным в пространстве $L_{2}(0,1)$. Ниже доказывается положительность оператора $P_{01}^{\varphi}$ с ядром $\varphi(x)$, удовлетворяющим менее жестким условиям леммы 2.1 (т.е. без предположения о неотрицательности и непрерывной дифференцируемости функции $\varphi(x))$ в более широких пространствах Лебега $L_{01}^{p}(\rho)$ с произвольным (не обязательно степенным) весом $\rho(x)$.

Определение 2.1. Скажем, что $\varphi(x) \in \Omega_{01}^{1}$, если $\varphi(x)$ - такая непрерывная невозрастающая выпуклая вниз в промежутке $(0,1]$ функция, что

$$
\int_{0}^{1} \varphi(x) d x \geqslant 0
$$

Теорема 2.2. Пусть $1<p<\infty$ и вес $\rho(x)$ удовлетворяет условиям (2.1) и (2.2). Если

$$
\begin{array}{ll}
\varphi(x) \in L_{01}^{p^{\prime} / 2} \cap \Omega_{01}^{1} & \text { при } 1<p \leqslant 2, \\
\varphi(x) \in L_{01}^{1} \cap \Omega_{01}^{1} & \text { при } 2<p<\infty,
\end{array}
$$

то оператор $P_{01}^{\varphi}$ действует непрерывно из пространства $L_{01}^{p}(\rho)$ в сопряэсенное с ним пространство $L_{01}^{p^{\prime}}\left(\rho^{1-p^{\prime}}\right), p^{\prime}=p /(p-1)$, и положителен, причем для любого $u(x) \in L_{01}^{p}(\rho)$ справедливо неравенство

$$
\left\langle P_{01}^{\varphi} u, u\right\rangle=\frac{a_{0}}{2}\left(\int_{0}^{1} u(x) d x\right)^{2}+\sum_{n=1}^{\infty} a_{n}\left|\int_{0}^{1} u(x) e^{i \pi n x} d x\right|^{2} \geqslant 0
$$

где

$$
a_{n}=2 \int_{0}^{1} \varphi(x) \cos (\pi n x) d x \geqslant 0, \quad n=0,1,2, \ldots
$$

Доказательство. Тот факт, что оператор $P_{01}^{\varphi}$ действует непрерывно из $L_{01}^{p}(\rho)$ в $L_{01}^{p^{\prime}}\left(\rho^{1-p^{\prime}}\right)$ вытекает из теоремы 2.1. Докажем его положительность (ср. [9]). Положим

$$
f_{\varepsilon}(x)= \begin{cases}\varphi(x+\varepsilon) & \text { при } 0 \leqslant x \leqslant 1-\varepsilon \\ \varphi(1) & \text { при } 1-\varepsilon<x \leqslant 1\end{cases}
$$

где $\varepsilon \in(0,1)$ - достаточно малое число. Очевидно, что функция $f_{\varepsilon}(x)$ удовлетворяет всем требованиям леммы 2.1 и $\lim _{\varepsilon \rightarrow 0} f_{\varepsilon}(x)=\varphi(x)$. Продолжим функцию $f_{\varepsilon}(x)$ на отрезок $[-1,0]$ четным образом. По теореме Дирихле имеем

$$
f_{\varepsilon}(x)=\frac{a_{0}^{\varepsilon}}{2}+\sum_{n=1}^{\infty} a_{n}^{\varepsilon} \cos (\pi n x), \quad 0<x<1,
$$

где

$$
a_{n}^{\varepsilon}=2 \int_{0}^{1} f_{\varepsilon}(x) \cos (\pi n x) d x .
$$


Так как $\varphi(x) \in \Omega_{01}^{1}$, то

$$
a_{0}^{0}=\lim _{\varepsilon \rightarrow 0} a_{0}^{\varepsilon}=\lim _{\varepsilon \rightarrow 0} 2 \int_{0}^{1} f_{\varepsilon}(x) d x=2 \int_{0}^{1} \varphi(x) d x \geqslant 0
$$

и, в силу неравенства $(2.12), a_{n}^{0}=\lim _{\varepsilon \rightarrow 0} a_{n}^{\varepsilon} \geqslant 0$ для всех $n \in \mathbb{N}$. Поэтому для любой функции $u(x) \in L_{01}^{p}(\rho)$ с учетом равенства $(2.16)$ имеем

$$
\begin{aligned}
\left\langle P_{01}^{\varphi} u, u\right\rangle=\int_{0}^{1} & \left(\int_{0}^{1} \varphi(|x-t|) u(t) d t\right) u(x) d x=\lim _{\varepsilon \rightarrow 0} \int_{0}^{1}\left(\int_{0}^{1} f_{\varepsilon}(|x-t|) u(t) d t\right) u(x) d x= \\
& =\lim _{\varepsilon \rightarrow 0} \int_{0}^{1}\left(\frac{a_{0}^{\varepsilon}}{2} \int_{0}^{1} u(t) d t+\sum_{n=1}^{\infty} a_{n}^{\varepsilon} \int_{0}^{1} \cos (\pi n|x-t|) u(t) d t\right) u(x) d x= \\
& =\frac{a_{0}^{0}}{2}\left(\int_{0}^{1} u(x) d x\right)^{2}+\sum_{n=1}^{\infty} a_{n}^{0}\left[\left(\int_{0}^{1} u(x) \cos (\pi n x) d x\right)^{2}+\left(\int_{0}^{1} u(x) \sin (\pi n x) d x\right)^{2}\right] .
\end{aligned}
$$

Следовательно,

$$
\left\langle P_{01}^{\varphi} u, u\right\rangle=\frac{a_{0}^{0}}{2}\left(\int_{0}^{1} u(x) d x\right)^{2}+\sum_{n=1}^{\infty} a_{n}^{0}\left|\int_{0}^{1} u(x) e^{i \pi n x} d x\right|^{2} \geqslant 0,
$$

т.е. оператор $P_{01}^{\varphi}$ является положительным и выполнено неравенство (2.14).

Аналогично доказывается следующая теорема, двойственная теореме 2.2 .

Теорема 2.3. Пусть $1<p<\infty$, вес $\rho(x)$ и ядро $\varphi(x)$ удовлетворяют условиям

$$
\begin{aligned}
\|\rho\|_{2 /(2-p)}^{1 / p}= & \left(\int_{0}^{1}[\rho(x)]^{2 /(2-p)} d x\right)^{(2-p) /(2 p)}<\infty, \quad \text { если } 1<p<2, \\
\|\rho\|_{\infty}^{1 / p}=\left[\underset{0 \leqslant x \leqslant 1}{\operatorname{ess} \sup \rho(x)]^{1 / p}<\infty,}\right. & \text { если } 2 \leqslant p<\infty, \\
& \varphi(x) \in L_{01}^{1} \cap \Omega_{01}^{1}, \quad \text { если } 1<p<2, \\
& \varphi(x) \in L_{01}^{p / 2} \cap \Omega_{01}^{1}, \quad \text { если } 2 \leqslant p<\infty .
\end{aligned}
$$

Тогда оператор $P_{01}^{\varphi}$ действует непрерывно из $L_{01}^{p^{\prime}}\left(\rho^{1-p^{\prime}}\right)$ в $L_{01}^{p}(\rho)$ и положителен, причем для любого $и(x) \in L_{01}^{p^{\prime}}\left(\rho^{1-p^{\prime}}\right)$ справедливо неравенство

$$
\left\|P_{01}^{\varphi} u\right\|_{p, 1} \leqslant C^{*} \cdot\|u\|_{p^{\prime}, 1-p^{\prime}}
$$

где

$$
C^{*}= \begin{cases}2\|\rho\|_{2 /(2-p)}^{2 / p}\|\varphi\|_{1}, & \text { если } 1<p<2, \\ 2^{2 / p}\|\rho\|_{\infty}^{2 / p}\|\varphi\|_{p / 2}, & \text { если } 2 \leqslant p<\infty .\end{cases}
$$

Замечание 2.2. Поскольку функции $\varphi(x)=x^{\alpha-1}$ при $0<\alpha<1$ и $\varphi(x)=-\ln x$ принадлежат классу $\Omega_{01}^{1}$, то теоремы 2.2 и 2.3 охватывают потенциал Рисса $I^{\alpha}$ и логарифмический потенциал (см. [1]). Поэтому $P_{01}^{\varphi}$ мы называем обобщенным оператором типа потенциала. 
3. Теоремы существования и единственности. Оценки решений. Используя теоремы 2.1-2.3, можно доказать теоремы существования и единственности решения для различных классов нелинейных интегральных уравнений, содержащих оператор $P_{01}^{\varphi}$.

Введем в рассмотрение нелинейный оператор суперпозиции (так называемый оператор Немыцкого). Пусть вещественнозначная функция $F(x, u)$ определена при $x \in[0,1], u \in \mathbb{R}$ и удовлетворяет условиям Каратеодори: она измерима по $x$ при каждом фиксированном $u \in \mathbb{R}$ и непрерывна по $u$ почти для всех $x \in[0,1]$. Обозначим через $F$ оператор суперпозиции, порожденный функцией $F(x, u)$, а через $L_{01}^{p,+}(\rho)$ - множество всех неотрицательных функций из $L_{01}^{p}(\rho)$.

Рассмотрим сначала нелинейное уравнение, наиболее простое для исследования методом монотонных операторов.

Теорема 3.1. Пусть $1<p<\infty, f(x) \in L_{01}^{p^{\prime}}\left(\rho^{1-p^{\prime}}\right)$, вес $\rho(x)$ и ядро $\varphi(x)$ удовлетворлют условиям (2.1), (2.2) и (2.13). Предположим, что для почти всех $x \in[0,1]$ и всех $u \in \mathbb{R}$ выполняются следуюшие условия:

(3.1) $|F(x, u)| \leqslant a(x)+d_{1} \cdot \rho(x) \cdot|u|^{p-1}$, где $a(x) \in L_{01}^{p^{\prime},+}\left(\rho^{1-p^{\prime}}\right), d_{1}>0$;

(3.2) $F(x, u)$ не убывает по и;

(3.3) $F(x, u) \cdot u \geqslant d_{2} \cdot \rho(x) \cdot|u|^{p}-D(x)$, где $D(x) \in L_{01}^{1,+}, d_{2}>0$.

Тогда при любых значениях параметра $\lambda>0$ уравнение

$$
\lambda \cdot F(x, u(x))+\int_{0}^{1} \varphi(|x-t|) \cdot u(t) d t=f(x)
$$

имеет решение $u^{*}(x) \in L_{01}^{p}(\rho)$. Это решение единственно, если в условии (3.2) фуункия $F(x, u)$ строго возрастает по и. Кроме того, если в условии (з.3) $D(x)=0$, то справедлива оченка:

$$
\left\|u^{*}\right\|_{p, 1} \leqslant\left(\lambda^{-1} d_{2}^{-1}\|f\|_{p^{\prime}, 1-p^{\prime}}\right)^{1 /(p-1)} .
$$

Доказательство. Запишем уравнение (3.1) в операторном виде:

$$
A u=f, \quad \text { где } A u=\lambda \cdot F u+P_{01}^{\varphi} u .
$$

Из условий (3.1)-(3.3) вытекает, соответственно, что оператор Немыцкого $F$, порожденный функцией $F(x, u)$, действует непрерывно из $L_{01}^{p}(\rho)$ в $L_{01}^{p^{\prime}}\left(\rho^{1-p^{\prime}}\right)$, монотонен и коэрцитивен, причем для всех $u(x) \in L_{01}^{p}(\rho)$ выполняются неравенства

$$
\|F u\|_{p^{\prime}, 1-p^{\prime}} \leqslant\|a\|_{p^{\prime}, 1-p^{\prime}}+d_{1} \cdot\|u\|_{p, 1}^{p-1}, \quad\langle F u, u\rangle \geqslant d_{2} \cdot\|u\|_{p, 1}^{p}-\|D\|_{1} .
$$

Из первого неравенства также вытекает, что нелинейный оператор $F$ является ограниченным.

Рассмотрим теперь оператор $P_{01}^{\varphi}$. По теореме 2.2 оператор $P_{01}^{\varphi}$ действует непрерывно из $L_{01}^{p}(\rho)$ в $L_{01}^{p^{\prime}}\left(\rho^{1-p^{\prime}}\right)$ и является положительным оператором. Таким образом, для оператора $A=P_{01}^{\varphi}+\lambda \cdot F$ выполняются все требования теоремы Браудера-Минти (см. [7]). Следовательно, уравнение (3.2), а значит и уравнение (3.1), имеет решение $u^{*}(x) \in L_{01}^{p}(\rho)$. Это решение единственно, если $F(x, u)$ в условии (3.2) строго возрастает по $u$, поскольку в этом случае оператор $A=P_{01}^{\varphi}+\lambda \cdot F$ будет строго монотонным.

Наконец, используя условие (3.3) при $D(x)=0$, положительность оператора $P_{01}^{\varphi}$ и равенство $A u^{*}=f$, имеем

$$
\lambda d_{2}\left\|u^{*}\right\|_{p, 1}^{p} \leqslant \lambda\left\langle F u^{*}, u^{*}\right\rangle \leqslant \lambda\left\langle F u^{*}, u^{*}\right\rangle+\left\langle P_{01}^{\varphi} u^{*}, u^{*}\right\rangle=\left\langle A u^{*}, u^{*}\right\rangle=\left\langle f, u^{*}\right\rangle \leqslant\|f\|_{p^{\prime}, 1-p^{\prime}}\left\|u^{*}\right\|_{p, 1},
$$

откуда непосредственно вытекает доказываемая оценка.

Прежде чем рассмотреть другой класс нелинейных уравнений с ядром типа потенциала, приведем один результат с поправками (см. ниже), установленный в [12], придерживаясь обозначений этой работы. Пусть $\Omega$-пространство с $\sigma$-конечной мерой, $K$-монотонный оператор и $(F u)(x)=f(x, u(x))$ - оператор Немыцкого. Рассмотрим уравнение типа Гаммерштейна

$$
u+K F u=g .
$$

Справедлива следующая теорема Брезиса-Браудера (ср. [12, теорема 3]). 
Теорема 3.2 (см. [12]). Пусть $p>1$ и $K$-монотонный, хеминепрерывный и ограниченный оператор из $L_{p^{\prime}}(\Omega)$ в $L_{p}(\Omega)$. Предположим, что функиия $f(x, r): \Omega \times \mathbb{R} \rightarrow \mathbb{R}$ непрерывна и не убывает по $r$ для почти всех $x \in \Omega$, является измеримой по $x$ для всех $r \in \mathbb{R}$ и удовлетворяет условию

$$
|f(x, r)| \leqslant c(x)+c_{0} \cdot|r|^{p-1} \quad \text { для почти всех } x \in \Omega \text { и всех } r \in \mathbb{R},
$$

где $c \in L_{p^{\prime}}(\Omega), c_{0}>0$. Тогда уравнение (3.3) имеет единственное решение $u \in L_{p}(\Omega)$ при любом $g \in L_{p}(\Omega)$.

Следует отметить, что в формулировке теоремы 3.2, приведенной в [12, с. 570], допущены неточности, а именно, функция $f(x, r)$ предполагается невозрастающей по $r$ (что не согласуется с условием (3) из [12, с. 567]) и измеримой по $x$ для всех $x \in \mathbb{R}$ (должно быть для всех $r \in \mathbb{R}$, что очевидно). Кроме того, пропущены требования, что $p>1$ и $c_{0}>0$. Покажем на простом примере, что если функция $f(x, r)$ является невозрастающей по $r$, то утверждение [12, теорема 3] о единственности решения неверно. Рассмотрим в пространстве $L_{4 / 3}(\Omega)$ уравнение

$$
u(x)-b(x) \int_{\Omega} b(s) \cdot u^{1 / 3}(s) d s=0,
$$

где заданная функция $b(x) \neq 0$ почти всюду на $\Omega$ и $b(x) \in L_{4 / 3}(\Omega)$. В данном случае, в соответствии с [12, теорема 3], $p=4 / 3, f(x, r)=-r^{1 / 3}$ является невозрастающей по $r$ функцией и оператор $K$ имеет вид

$$
(K u)(x)=b(x) \int_{\Omega} b(s) u(s) d s .
$$

Так как для любого $u(x) \in L_{4}(\Omega)$ имеем

$$
\|K u\|_{4 / 3}=\left|\int_{\Omega} b(s) u(s) d s\right| \cdot\left(\int_{\Omega}|b(x)|^{4 / 3} d x\right)^{3 / 4} \leqslant\|b\|_{4 / 3}^{2} \cdot\|u\|_{4},
$$

то оператор $K$ действует из пространства $L_{4}(\Omega)$ в сопряженное с ним пространство $L_{4 / 3}(\Omega)$ и ограничен. Поскольку $K$ является линейным оператором, то он является также непрерывным и тем более хеминепрерывным оператором. Далее, для любого $u(x) \in L_{4}(\Omega)$ имеем

$$
\langle K u, u\rangle=\int_{\Omega}\left(b(x) \int_{\Omega} b(s) u(s) d s\right) u(x) d x=\left(\int_{\Omega} b(s) u(s) d s\right)^{2} \geqslant 0,
$$

т.е. $K$ - положительный, а значит, в силу своей линейности, и монотонный оператор. Таким образом, оператор $K$ и функция $f(x, r)=-r^{1 / 3}$ удовлетворяют всем требованиям [12, теорема 3], при $g=0, c(x)=0, c_{0}=1$ и $p=4 / 3$.

Покажем, наконец, что уравнение (3.4) имеет два различных решения в пространстве $L_{4 / 3}(\Omega)$. Пусть $u(x) \in L_{4 / 3}(\Omega)$ есть любое нетривиальное, т.е. отличное от $u(x)=0$, решение уравнения (3.4). Положим

$$
\int_{\Omega} b(s) u^{1 / 3}(s) d s=C .
$$

Тогда из (3.4) получаем:

$$
u(x)=C \cdot b(x) \quad \text { или } \quad b(x) \cdot u^{1 / 3}(x)=C^{1 / 3} b^{4 / 3}(x) .
$$

Интегрируя последнее равенство, имеем

$$
C=C^{1 / 3} \int_{\Omega} b^{4 / 3}(x) d x \quad \text { или } \quad C=\left(\int_{\Omega} b^{4 / 3}(x) d x\right)^{3 / 2} .
$$


Подставляя найденное значение $C$ в первое равенство из (3.5), окончательно получаем

$$
u(x)=\left(\int_{\Omega} b^{4 / 3}(s) d s\right)^{3 / 2} b(x) .
$$

Таким образом, уравнение (3.4) помимо тривиального решения $u(x)=0$ имеет еще и нетривиальное решение (3.6) в пространстве $L_{4 / 3}(\Omega)$, что противоречит утверждению о единственности решения в формулировке [12, теорема 3].

Замечание 3.1. В [13, с. 126] доказан более общий, чем в теореме 3.2, результат, из которого, в частности, следует, что в формулировке [12, теорема 3] функция $f(x, r)$ должна не убывать по $r$ и при этом она справедлива и для весовых пространств $L_{01}^{p}(\rho)$.

В отличие от теоремы 3.1, в следующей теореме существование и единственность решения доказывается без условия корцитивности (3.3) нелинейности $F(x, u)$, причем на вес $\rho(x)$ и ядро $\varphi(x)$ накладываются другие условия.

Теорема 3.3. Пусть $1<p<\infty, f(x) \in L_{01}^{p}(\rho)$, вес $\rho(x)$ и ядро $\varphi(x)$ удовлетворяют условиям (2.17), (2.18) и (2.19). Если нелинейность $F(x, u)$ удовлетворяет условиям (3.1) и (3.2), то при любых $\lambda \geqslant 0$ уравнение

$$
u(x)+\lambda \cdot \int_{0}^{1} \varphi(|x-t|) \cdot F(t, u(t)) d t=f(x)
$$

имеет единственное решение $u^{*}(x) \in L_{01}^{p}(\rho)$. Кроме того, если выполнены условия (3.1) и (3.3) при с $(x)=D(x)=0$, то справедлива оценка

$$
\left\|u^{*}\right\|_{p, 1} \leqslant d_{1} d_{2}^{-1}\|f\|_{p, 1} .
$$

Доказательство. При $\lambda=0$ утверждения теоремы очевидны, поэтому считаем далее, что $\lambda>0$. Запишем уравнение (3.7) в операторном виде:

$$
u+\lambda \cdot P_{01}^{\varphi} F u=f .
$$

Из условий (3.1) и (3.2) вытекает, что оператор $F$ действует непрерывно из $L_{01}^{p}(\rho)$ в $L_{01}^{p^{\prime}}\left(\rho^{1-p^{\prime}}\right)$ и является монотонным, а из условия $(2.19)$, в силу теоремы 2.3 , вытекает, что оператор $P_{01}^{\varphi}$ действует непрерывно из $L_{01}^{p^{\prime}}\left(\rho^{1-p^{\prime}}\right)$ обратно в $L_{01}^{p}(\rho)$ и положителен. Но тогда, в силу теоремы 3.2 и замечания 3.1, данное уравнение имеет единственное решение $u^{*}(x) \in L_{01}^{p}(\rho)$.

Осталось доказать оценку нормы решения $u^{*}(x)$. Используя условия (3.1) и (3.3) при $c(x)=$ $D(x)=0$, положительность оператора $P_{01}^{\varphi}$ и равенство $u^{*}+\lambda \cdot P_{01}^{\varphi} F u^{*}=f$, имеем

$$
d_{2}\left\|u^{*}\right\|_{p, 1}^{p} \leqslant\left\langle u^{*}, F u^{*}\right\rangle+\lambda\left\langle P_{01}^{\varphi} F u^{*}, F u^{*}\right\rangle=\left\langle f, F u^{*}\right\rangle \leqslant\|f\|_{p, 1}\left\|F u^{*}\right\|_{p^{\prime}, 1-p^{\prime}} \leqslant d_{1}\|f\|_{p, 1}\left\|u^{*}\right\|_{p, 1}^{p-1},
$$

откуда непосредственно вытекает доказываемая оценка.

Следующая теорема отличается от теорем 3.1 и 3.3 как по характеру ограничений на нелинейность $F(x, u)$ и правую часть $f(x)$, так и по структуре доказательства, основанном на обращении нелинейного оператора суперпозиции и установлении коэрцитивности обратного оператора.

Теорема 3.4. Пусть $1<p<\infty, f(x) \in L_{01}^{p}(\rho)$, вес $\rho(x)$ и ядро $\varphi(x)$ удовлетворяют условиям (2.1), (2.2) и (2.13). Предположим, что для почти всех $x \in[0,1]$ и всех $u \in \mathbb{R}$ выполняются следующие условия:

(3.4) $|F(x, u)| \leqslant g(x)+d_{3} \cdot\left([\rho(x)]^{-1}|u|\right)^{1 /(p-1)}$, где $g(x) \in L_{01}^{p,+}(\rho), d_{3}>0$;

(3.5) $F(x, u)$ строго возрастает по $u$;

(3.6) $F(x, u) \cdot u \geqslant d_{4} \cdot\left([\rho(x)]^{-1}|u|\right)^{1 /(p-1)}|u|-D(x)$, где $D(x) \in L_{01}^{1,+}, d_{4}>0$. 
Тогда о при любых значениях параметра $\lambda \geqslant 0$ уравнение

$$
u(x)+\lambda \cdot F\left(x, \int_{0}^{1} \varphi(|x-t|) \cdot u(t) d t\right)=f(x)
$$

имеет единственное решение $u^{*}(x) \in L_{01}^{p}(\rho)$. Кроме того, если условия (3.4) и (3.6) выполненъ при $g(x)=D(x)=0$, то справедлива оченка

$$
\left\|u^{*}-f\right\|_{p, 1} \leqslant \lambda\left(d_{3}^{p} d_{4}^{-1} C\|f\|_{p, 1}\right)^{1 /(p-1)}
$$

где константа C определено в (2.4).

Доказательство. При $\lambda=0$ утверждение данной теоремы очевидно, поэтому считаем далее, что $\lambda>0$. Запишем уравнение $(3.8)$ в операторном виде:

$$
u+\lambda \cdot F P_{01}^{\varphi} u=f
$$

и покажем, что оно имеет решение $u^{*}(x) \in L_{01}^{p}(\rho)$. Для этого введем новую неизвестную функцию $v(x)$, положив $f(x)-u(x)=\lambda \cdot v(x)$. Ясно, что $v(x) \in L_{01}^{p}(\rho)$. Подставив $u=f-\lambda \cdot v$ в уравнение (3.9), получим:

$$
F P_{01}(f-\lambda \cdot v)=v
$$

Из условий (3.4)-(3.6) вытекает, что оператор Немыцкого $F$ действует непрерывно из сопряженного пространства $L_{01}^{p^{\prime}}\left(\rho^{1-p^{\prime}}\right)$ в исходное пространство $L_{01}^{p}(\rho)$, строго монотонен и коэрцитивен, причем для любого $w(x) \in L_{01}^{p^{\prime}}\left(\rho^{1-p^{\prime}}\right)$ выполняются неравенства

$$
\|F w\|_{p, 1} \leqslant\|g\|_{p, 1}+d_{3}\|w\|_{p^{\prime}, 1-p^{\prime}}^{1 /(p-1)}, \quad\langle F w, w\rangle \geqslant d_{4}\|w\|_{p^{\prime}, 1-p^{\prime}}^{p /(p-1)}-\|D\|_{1} .
$$

Значит, согласно [7, теорема 2.2] существует обратный оператор $F^{-1}$, действующий из $L_{01}^{p}(\rho)$ в $L_{01}^{p^{\prime}}\left(\rho^{1-p^{\prime}}\right)$ и являющийся строго монотонным, ограниченным и радиально непрерывным (заметим, что для монотонных операторов понятия радиально непрерывный и деминепрерывный совпадают в силу [7, лемма 1.3]).

Покажем, что обратный оператор $F^{-1}$ является коэрцитивным, т.е. удовлетворяет условию

$$
\lim _{\|v\|_{p, 1} \rightarrow \infty} \frac{\left\langle F^{-1} v, v\right\rangle}{\|v\|_{p, 1}}=\infty .
$$

Пусть $v(x) \in L_{01}^{p}(\rho)$ и $F^{-1} v=w$. Тогда в силу первого неравенства из (3.11) имеем

$$
\|v\|_{p, 1}=\|F w\|_{p, 1} \leqslant\|g\|_{p, 1}+d_{3}\|w\|_{p^{\prime}, 1-p^{\prime}}^{1 /(p-1)}
$$

Следовательно, $\|w\|_{p^{\prime}, 1-p^{\prime}} \rightarrow \infty$, если $\|v\|_{p, 1} \rightarrow \infty$. Поэтому, используя неравенство (3.13) и второе неравенство из (3.11), получаем

$$
\frac{\left\langle F^{-1} v, v\right\rangle}{\|v\|_{p, 1}}=\frac{\langle w, F w\rangle}{\|v\|_{p, 1}} \geqslant \frac{d_{4}\|w\|_{p^{\prime}, 1-p^{\prime}}^{p /(p-1)}-\|D\|_{1}}{\|g\|_{p, 1}+d_{3}\|w\|_{p^{\prime}, 1-p^{\prime}}^{1 /(p-1)}} \rightarrow \infty \quad \text { при }\|v\|_{p, 1} \rightarrow \infty,
$$

т.е. справедливо равенство (3.12), и поэтому оператор $F^{-1}$ является коэрцитивным.

Применив теперь оператор $F^{-1}$ к обеим частям уравнения $(3.10)$, имеем $P_{01}^{\varphi} f-\lambda \cdot P_{01}^{\varphi} v=F^{-1} v$ или

$$
F^{-1} v+\lambda \cdot P_{01}^{\varphi} v=P_{01}^{\varphi} f
$$

т.е. приходим к уравнению вида (3.2).

Поскольку оператор $A=F^{-1}+\lambda \cdot P_{01}^{\varphi}$ удовлетворяет всем требованиям теоремы БраудераМинти, то уравнение (3.15) имеет решение $v^{*} \in L_{01}^{p}(\rho)$. Единственность этого решения вытекает из строгой монотонности оператора $F^{-1}$. Но тогда, в силу связи $u=f-\lambda \cdot v$, уравнение $(3.9)$, а значит и данное уравнение (3.8), имеет единственное решение $u^{*} \in L_{01}^{p}(\rho)$. 
Осталось доказать оценку нормы решения. Положим $\psi=F^{-1} v^{*}$. Тогда $F \psi=v^{*}$. Так как $F^{-1} v^{*}+\lambda \cdot P_{01}^{\varphi} v^{*}=P_{01}^{\varphi} f$, то с учетом теоремы 2.2 и равенств $g(x)=D(x)=0$ имеем

$$
\begin{aligned}
d_{4}\|\psi\|_{p^{\prime}, 1-p^{\prime}}^{p^{\prime}} \leqslant\langle F \psi, \psi\rangle=\left\langle v^{*}, F^{-1} v^{*}\right\rangle & \leqslant\left\langle v^{*}, F^{-1} v^{*}\right\rangle+\lambda\left\langle v^{*}, P_{01}^{\varphi} v^{*}\right\rangle=\left\langle F \psi, P_{01}^{\varphi} f\right\rangle \leqslant \\
& \leqslant\|F \psi\|_{p, 1}\left\|P_{01}^{\varphi} f\right\|_{p^{\prime}, 1-p^{\prime}} \leqslant C\|F \psi\|_{p, 1}\|f\|_{p, 1} \leqslant C d_{3}\|\psi\|_{p^{\prime}, 1-p^{\prime}}^{p^{\prime}-1}\|f\|_{p, 1} .
\end{aligned}
$$

Следовательно,

Так как

$$
\|\psi\|_{p^{\prime}, 1-p^{\prime}} \leqslant d_{3} d_{4}^{-1} C\|f\|_{p, 1} .
$$

TO

$$
\left\|v^{*}\right\|_{p, 1}=\|F \psi\|_{p, 1} \leqslant d_{3}\|\psi\|_{p^{\prime}, 1-p^{\prime}}^{p^{\prime}-1}, \quad v^{*}=\lambda^{-1}\left(f-u^{*}\right)
$$

$$
\left\|f-u^{*}\right\|_{p, 1} \leqslant \lambda d_{3}\|\psi\|_{p^{\prime}, 1-p^{\prime}}^{1 /(p-1)}
$$

откуда с учетом неравенства (3.16) получаем доказываемую оценку нормы решения.

Замечание 3.2. Из оценок для норм решений, полученных в теоремах 3.1, 3.3 и 3.4, непосредственно вытекает, что уравнения (3.1), (3.7) и (3.8) при $f(x)=0$ имеют лишь тривиальное решение $u(x)=0$.

В заключение отметим, что при $\rho(x)=1$ аналоги теорем $3.1,3.2$ и 3.4 были доказаны в [3]. Вопрос о приближенном решении уравнений $(3.1),(3.7)$ и (3.8) в гильбертовом пространстве $L_{01}^{2}$ был рассмотрен в [5], где комбинированием метода монотонных операторов и принципа сжимающих отображений показано, что решения этих уравнений могут быть найдены методом последовательных приближений пикаровского типа.

\section{СПИСОК ЛИТЕРАТУРЫ}

1. Асхабов C. Н. Нелинейные уравнения типа свертки. - М.: Физматлит, 2009.

2. Асхабов C. Н. Уравнения типа свертки с монотонной нелинейностью на отрезке// Диффер. уравн. 2015. - 51, № 9. - С. 1182-1188.

3. Асхабов С. Н. Нелинейные уравнения типа свертки в пространствах Лебега// Мат. заметки. - 2015. -97, № 5 . - С. 643-654.

4. Асхабов C. Н. Условия положительности операторов с разностными ядрами в рефлексивных пространствах // Итоги науки и техн. Совр. мат. прилож. Темат. обзоры. - 2018. - 149. - С. 3-13.

5. Асхабов С. Н., Джабраилов А. Л. Приближенное решение нелинейных уравнений типа свертки на отрезке// Уфим. мат. ж. - 2013. - 5, № 2. - С. 3-11.

6. Вайнберг M. М. Вариационный метод и метод монотонных операторов в теории нелинейных уравнений. - М.: Наука, 1972.

7. Гаевский X., Грегер K., Захариас K. Нелинейные операторные уравнения и операторные дифференциальные уравнения. - М.: Мир, 1978.

8. Гахов Ф. Д., Черский Ю. И. Уравнения типа свертки. - М.: Наука, 1978.

9. Нахушев А. М. Дробное исчисление и его применение. - М.: Физматлит, 2003.

10. Харди Г. Х., Рогозинский В. В. Ряды Фурье. - М.: Физматгиз, 1959.

11. Эдвардс Р. Ряды Фурье в современном изложении. - М.: Мир, 1985.

12. Brezis H., Browder F. E. Some new results about Hammerstein equations// Bull. Am. Math. Soc. - 1974. - 80. - P. 567-572.

13. Brezis H., Browder F. E. Nonlinear integral equations and systems of Hammerstein type// Adv. Math. 1975. - 18. - P. 115-147.

14. Brunner H. Volterra Integral Equations: An Introduction to Theory and Applications. - Cambridge: Cambridge Univ. Press, 2017.

15. Gripenberg G., Londen S. O., Staffans O. Volterra Integral and Functional Equations. - Cambridge-New York: Cambridge Univ. Press, 1990.

Асхабов Султан Нажмудинович

Чеченский государственный педагогический университет, Грозный;

Чеченский государственный университет, Грозный

E-mail: askhabov@yandex.ru 